\title{
Resveratrol improves insulin sensitivity, reduces oxidative stress and activates the Akt pathway in type 2 diabetic patients
}

\author{
Pál Brasnyó ${ }^{1}$, Gergő A. Molnár ${ }^{1}$, Márton Mohás ${ }^{1}$, Lajos Markó ${ }^{1}$, Boglárka Laczy ${ }^{1}$, Judit Cseh $^{1}$, \\ Esztella Mikolás ${ }^{1}$, István András Szijártó ${ }^{1}$, Ákos Mérei ${ }^{1}$, Richárd Halmai ${ }^{1}$, László G. Mészáros ${ }^{2}$, \\ Balázs Sümegi ${ }^{3}$ and István Wittmann ${ }^{1 *}$ \\ ${ }^{1}$ 2nd Department of Internal Medicine and Nephrological Center, Faculty of Medicine, University of Pécs, Pacsirta Street 1, \\ Pécs H-7624, Hungary \\ ${ }^{2}$ Life Sciences Foundation, Theoretical Biochemistry Group, Augusta, GA 30909, USA \\ ${ }^{3}$ Department of Biochemistry and Medical Chemistry, Faculty of Medicine, University of Pécs, Pécs H-7624, Hungary
}

(Received 25 August 2010 - Revised 7 December 2010 - Accepted 17 January 2011 - First published online 9 March 2011)

\section{Abstract}

Although resveratrol has widely been studied for its potential health benefits, little is known about its metabolic effects in humans. Our aims were to determine whether the polyphenol resveratrol improves insulin sensitivity in type 2 diabetic patients and to gain some insight into the mechanism of its action. After an initial general examination (including blood chemistry), nineteen patients enrolled in the 4-weeklong double-blind study were randomly assigned into two groups: a resveratrol group receiving oral $2 \times 5 \mathrm{mg}$ resveratrol and a control group receiving placebo. Before and after the second and fourth weeks of the trial, insulin resistance/sensitivity, creatinine-normalised ortho-tyrosine level in urine samples (as a measure of oxidative stress), incretin levels and phosphorylated protein kinase B (pAkt):protein kinase B (Akt) ratio in platelets were assessed and statistically analysed. After the fourth week, resveratrol significantly decreased insulin resistance (homeostasis model of assessment for insulin resistance) and urinary ortho-tyrosine excretion, while it increased the pAkt:Akt ratio in platelets. On the other hand, it had no effect on parameters that relate to $\beta$-cell function (i.e. homeostasis model of assessment of $\beta$-cell function). The present study shows for the first time that resveratrol improves insulin sensitivity in humans, which might be due to a resveratrol-induced decrease in oxidative stress that leads to a more efficient insulin signalling via the Akt pathway.

\section{Key words: Resveratrol: Type 2 diabetes: Insulin sensitivity: Oxidative stress: Akt}

Despite the rather high average daily fat intake in France, compared with other European countries, epidemiological surveys document a relatively low rate of cardiovascular mortality. This phenomenon, which is often called the French paradox, is thought to be explained by a fairly high red wine consumption by the French ${ }^{(1,2)}$. Red wine is known to be rich in various polyphenolic compounds that might have a variety of health benefits. Among these polyphenols, the stilbene derivative resveratrol seems to be the most vigorously studied, which is probably due to the fact that it apparently affects a wide array of physiological and biochemical processes as shown in animal and cell culture studies ${ }^{(3)}$. On the other hand, human studies with conclusive results on resveratrol are regrettably lacking.
Resveratrol is considered to have beneficial effects on the cardiovascular system, as it has been found to improve vasodilatation $^{(4)}$, ischaemic preconditioning ${ }^{(5,6)}$, both of which seem to be the result of the activation of the endothelial NO synthase enzyme ${ }^{(7)}$, and to inhibit both platelet aggregation $^{(3)}$ and vascular smooth muscle cell proliferation ${ }^{(8)}$. In addition, resveratrol has also been demonstrated to show anti-inflammatory $^{(9)}$ and anti-tumour activities $^{(3)}$, and it might even have considerable anti-ageing properties as it provokes changes in cell signalling that mimics those found upon energy restriction ${ }^{(3)}$.

Oxidative stress, which is the result of either the overproduction of free radicals or some impairment in the cellular antioxidant systems, can lead to serious deterioration of

Abbreviations: Akt, protein kinase B; CGM, continuous glucose monitoring; GIP, glucose-dependent insulinotropic peptide; GLP-1, glucagon-like peptide 1; $\mathrm{HOMA}_{\beta}$, homeostasis model of assessment of $\beta$-cell function; HOMA $\mathrm{IR}$, homeostasis model of assessment for insulin resistance; ISI, insulin sensitivity index; MCR, metabolic clearance rate; o-Tyr, ortho-tyrosine; pAkt, phosphorylated protein kinase B.

*Corresponding author: Professor I. Wittmann, fax +36 72536 051, email istvan.wittmann@aok.pte.hu 
health. For instance, oxidative stress is likely to be involved in both the development and the advancement of diabetes mellitus, as several studies have demonstrated the involvement of oxygen free radicals (reactive oxygen species) in the appearance of insulin resistance, a distinctive characteristic of type 2 diabetes $^{(10-12)}$. In addition, oxidative stress might even play some direct role in the subsequent surfacing of other diabetes-related complications ${ }^{(13,14)}$

Resveratrol itself is an efficient antioxidant, as evidenced by both in vitro ${ }^{(15)}$ and in vivo studies ${ }^{(16)}$, and, in parallel, it has also been shown to improve diabetes-related impairments in animals ${ }^{(17,18)}$. Thus, in the present study, we address the question whether resveratrol has beneficial effects in controlling and/or improving insulin resistance also in humans, and at the same time, whether it alters oxidative status. In addition, we also aim to elucidate the underlying biochemical mechanisms that might - at least in part - explain the effects of resveratrol, especially in relation to type 2 diabetes.

\section{Patients and methods}

\section{Patients}

The present study was conducted according to the guidelines laid down in the Declaration of Helsinki, and all procedures involving human patients were approved by the Research Ethics Committee, University of Pécs (Pécs, Hungary; certificate no. 2168). Written informed consent was obtained from all participating patients.

A total of nineteen Caucasian male patients previously diagnosed with type 2 diabetes (according to the WHO diagnostic guidelines) were included in the study. They underwent a blinded randomisation into two groups: ten patients to receive oral resveratrol twice daily (in gelatin capsules containing $5 \mathrm{mg}$ resveratrol) and nine patients to placebo (two capsules daily, see also below). All nineteen patients were over 18 years of age, had normal creatinine clearance $\geq 90 \mathrm{ml} / \mathrm{min}$ and were on either angiotensin-converting enzyme inhibitor or angiotensin II receptor blocker medication. Exclusion criteria were receiving insulin treatment, receiving corticosteroids, alcohol or drug abuse, severe liver or cardiac (New York Heart Association III-IV) disease, existing autoimmune disease, acute infection and any type of malignancy. The patients were instructed to abstain from any alcoholic beverages and foods containing substantial amounts of resveratrol (e.g. wine, red grapes, peanuts and berries). Compliance with these instructions was confirmed by verbal declaration of patients.

\section{Study protocol}

Before the initiation of the trial, all participants went through a general examination including past medical history, general physical survey, electrocardiogram, blood pressure, pulse rate and blood chemistry analysis (serum $\mathrm{Na}, \mathrm{K}$, urea, creatinine, total protein, albumin, $\mathrm{HbA}_{1 \mathrm{c}}$, fructosamine, alanine transaminase, aspartate aminotransferase, alkaline phosphatase, lactate dehydrogenase, $\gamma$-glutamyl transferase, bilirubin and prothrombin). The general examination was followed by a 4 -week washout period before the trial began (during which all lipid-lowering medication was ceased).

The day before the initiation of the trial (designated as baseline), $24 \mathrm{~h}$ urine samples from all patients were collected, from which creatinine, albumin and ortho-tyrosine (o-Tyr) concentrations were measured. Estimated glomerular filtration rate values were computed according to the Cockroft-Gault equation $^{(19)}$. During the same day, $24 \mathrm{~h}$ blood pressure (ambulatory blood pressure monitor) and a $48 \mathrm{~h}$ continuous tissue glucose monitoring (CGM; Medtronic MINIMED, SOFSENSOR, MMT-7002) were initiated. This was followed by drawing blood to measure glucose, insulin, C-peptide, TAG, LDL, HDL, total cholesterol, fructosamine, high-sensitivity C-reactive protein, fibrinogen, homocysteine, general blood chemistry (see also above), erythropoietin from the plasma and phosphorylated protein kinase B (pAkt):protein kinase $\mathrm{B}$ (Akt) ratio in platelets (prepared from the peripheral blood).

During the study period, the aforementioned measurements were repeated twice: at the end of the second (week 2) and the fourth weeks (week 4). After initiation of the CGM, the patients received a test meal (one portion, see later), which was also followed by the collection of blood samples to determine plasma glucose, serum insulin, C-peptide and TAG at 30, 60, 90 and $120 \mathrm{~min}$. In addition, urine was collected to measure $4 \mathrm{~h}$ creatinine clearance and albumin secretion.

\section{Analytical procedures}

Routine blood and urine tests were carried out by the Department of Laboratory Medicine at the University of Pécs Medical School according to standard clinical laboratory procedures. The values of homeostasis model of assessment for insulin resistance and related to $\beta$-cell function $\left(\mathrm{HOMA}_{\mathrm{IR}}\right.$ and $\mathrm{HOMA}_{\beta}$, respectively) were calculated as in Nagaretani et $a l .{ }^{(20)}$ and Matthews et al. ${ }^{(21)}$, respectively. Insulin sensitivity index (ISI Stumvoll $_{\text {) }}$ and glucose metabolic clearance rate $\left(\mathrm{MCR}_{\text {Stumvoll }}\right)$ were calculated as described previously ${ }^{(22)}$.

Oxidative stress was quantified by determining urinary $O-$ Tyr levels using a reverse-phase HPLC (Shimadzu LC-10 ADVP HPLC system; Shimadzu USA, Canby, OR, USA) equipped with a fluorescence detector (Shimadzu RF-10 AXL) as described previously ${ }^{(23)}$. Tyr and its isomers were excited at $275 \mathrm{~nm}$, and their emission was measured at $305 \mathrm{~nm}$. Urinary amino acid level was normalised to urinary creatinine concentration.

Platelets were isolated from peripheral blood samples as follows: to obtain washed human platelets, whole blood was collected from fasted (10-12 h) patients and was mixed with trisodium citrate $(9: 1 ; 130 \mathrm{~mm})$. Then, the citrated blood was centrifuged at $250 \boldsymbol{g}$ for $10 \mathrm{~min}$ to obtain the platelet-rich plasma by saving the supernatant into a separated polypropylene tube. The resultant platelet-rich plasma was mixed with an equal volume of washing buffer ( $\mathrm{pH} 7 \cdot 4)$ containing $20 \mathrm{~mm}$-Tris- $\mathrm{HCl}$ and $150 \mathrm{~mm}-\mathrm{NaCl}$. The platelet-rich plasma was subsequently centrifuged at $500 \mathrm{~g}$ for $10 \mathrm{~min}$, and the platelet pellet was suspended in HEPES-Tyrode's buffer ( $\mathrm{pH} 7 \cdot 4$ )

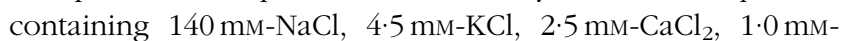
$\mathrm{MgCl}_{2}, 11 \mathrm{~mm}$-glucose and $20 \mathrm{~mm}$-HEPES, and was adjusted 
to a final concentration of $5.0 \times 10^{8}$ platelets $/ \mathrm{ml}$. Isolated platelets were lysed on ice for $30 \mathrm{~min}$ in NP40 buffer containing $20 \mathrm{~mm}$-Tris-HCl, $137 \mathrm{~mm}-\mathrm{NaCl}, \mathrm{NP} 40$ (1\%), glycerol (5\%) and $1.0 \mathrm{mm-EDTA}$, supplemented with phosphatase and protein kinase inhibitors containing $1.0 \mathrm{~mm}$-phenylmethylsulfonyl fluoride, 2.0 mm-sodium fluoride, $10 \mathrm{~mm}$-tetrasodium pyrophosphate, $2.0 \mathrm{~mm}$-sodium orthovanadate, $25 \mathrm{~mm}$-aprotinin and 25 mm-leupeptin. Protein concentration of the platelet lysates was determined by the Bradford assay (Bio-Rad, Hercules, CA, USA) using bovine serum albumin as standard. All chemicals were purchased from Sigma (St Louis, MO, USA).

The ratio of the phosphorylated form of Akt and total Akt (pAkt (Ser473):Akt) was determined by Western blot as described previously ${ }^{(24)}$.

Glucagon-like peptide 1 (GLP-1), glucose-dependent insulinotropic peptide (GIP) and amylin were quantified using ELISA kits commercially available from Millipore (Billerica, MA, USA): EGLP-35K for GLP-1; EZHGIP-54K for human GIP; EZHAT-51K for human amylin.

\section{Materials}

Resveratrol of herbal origin (with $>98 \% t$-resveratrol content) and the placebo (both in gelatin capsules) were obtained from Argina Nutraceuticals (previously Admarc Nutraceuticals, Fót, Hungary) and dosed $5 \mathrm{mg}$ /capsule. The identical placebo capsules contained only the carrier microcrystalline cellulose. A known number of (either test or placebo) capsules were boxed to check patient compliance at the end of the trial. The protein, carbohydrate and fat contents of the capsules (Mean values and standard deviations) were negligible. During the study, adverse effects of resveratrol or any signs of drug interaction have not been observed.

The test meal (Diben) was purchased from Fresenius Kabi (Bad Homburg, Germany). It was given to patients in $225 \mathrm{ml}$ portions; one portion had an energy content of $945 \mathrm{~kJ}$ from $10 \cdot 13 \mathrm{~g}$ protein, $20 \cdot 81 \mathrm{~g}$ carbohydrate and $11.25 \mathrm{~g}$ fat.

\section{Statistical analysis}

The measured parameters (CGM data, time until maximal interstitial glucose level, HOMA ${ }_{I R}$ values, o-Tyr:urinary creatinine ratio and pAkt:Akt ratio) showed normal distribution. The data from patients of the placebo and resveratrol groups were arranged respectively and evaluated by employing ANOVA using the program Origin (Microcal, Northampton, MA, USA), and are presented as means and standard deviations. Correlation between clinical parameters was analysed by Pearson's parametric correlation test using the program SPSS 13.0 for Windows (SPSS, Chicago, IL, USA).

As after the first 2 weeks into the trial no significant differences (for any of the measured parameters) between the two groups (i.e. resveratrol $v$. placebo) were observed, the data of week 2 are not shown. Because of the very large individual variations even at baseline in the HOMA IR and $o$-Tyr values within the same group, both measures were analysed on the individual level, i.e. for each participant, the value measured at baseline was subtracted from that measured at week 4 (i.e. $\Delta \mathrm{HOMA}_{\mathrm{IR}}$ and $\Delta$ o-Tyr:creatinine ratio), and then the resulting values were averaged within the respective groups.

Table 1. Clinical parameters at baseline of patients in the resveratrol and placebo groups*

\begin{tabular}{|c|c|c|c|c|}
\hline & \multicolumn{2}{|c|}{ Resveratrol (n 10) } & \multicolumn{2}{|c|}{ Placebo ( $n 9)$} \\
\hline & Mean & $\mathrm{SD}$ & Mean & SD \\
\hline Age (years) & $57 \cdot 9$ & $7 \cdot 9$ & $52 \cdot 5$ & $11 \cdot 1$ \\
\hline Body weight $(\mathrm{kg})$ & $90 \cdot 1$ & $16 \cdot 3$ & $105 \cdot 3$ & $16 \cdot 7$ \\
\hline High-sensitivity CRP (mg/l) & 3.35 & $2 \cdot 34$ & 4.05 & $1 \cdot 72$ \\
\hline Serum glucose $(\mathrm{mmol} / \mathrm{l})$ & 7.9 & $2 \cdot 21$ & $8 \cdot 8$ & $3 \cdot 2$ \\
\hline Fructosamine $(\mu \mathrm{mol} / \mathrm{l})$ & 281 & 44 & 288 & 44 \\
\hline $\mathrm{HbA}_{1 \mathrm{c}}(\%)$ & $7 \cdot 50$ & $2 \cdot 18$ & 7.65 & $1 \cdot 79$ \\
\hline Total cholesterol (mmol/l) & $5 \cdot 77$ & $1 \cdot 10$ & $5 \cdot 10$ & $1 \cdot 20$ \\
\hline LDL-cholesterol (mmol/l') & 3.40 & 1.03 & $3 \cdot 29$ & 1.08 \\
\hline HDL-cholesterol (mmol/l) & $1 \cdot 17$ & 0.23 & 0.97 & $0 \cdot 12$ \\
\hline $\mathrm{TAG}(\mathrm{mmol} / \mathrm{l})$ & $3 \cdot 20$ & 3.32 & 2.77 & $1 \cdot 31$ \\
\hline eGFR† (ml/min) & 117 & 30 & 138 & 38 \\
\hline Urinary albumin:creatinine (mg/mmol) & 4.07 & $4 \cdot 35$ & 1.95 & 3.03 \\
\hline Systolic blood pressure $(\mathrm{mmHg})$ & 140 & 12 & 140 & 17 \\
\hline Diastolic blood pressure (mmHg) & 86 & 5 & 89 & 12 \\
\hline \multicolumn{5}{|l|}{ Ratio of cases (yes/no) } \\
\hline Diabetic nephropathy & \multicolumn{2}{|c|}{$7 / 3$} & \multicolumn{2}{|c|}{$4 / 5$} \\
\hline Diabetic neuropathy & \multicolumn{2}{|c|}{$0 / 10$} & \multicolumn{2}{|c|}{$2 / 7$} \\
\hline Peripheric arterial disease & \multicolumn{2}{|c|}{$1 / 9$} & \multicolumn{2}{|c|}{$1 / 8$} \\
\hline Angina pectoris & \multicolumn{2}{|c|}{$0 / 10$} & \multicolumn{2}{|c|}{$1 / 8$} \\
\hline IHD & \multicolumn{2}{|c|}{$1 / 9$} & \multicolumn{2}{|c|}{$1 / 8$} \\
\hline Heart attack & \multicolumn{2}{|c|}{$0 / 10$} & \multicolumn{2}{|c|}{$0 / 9$} \\
\hline Stroke & \multicolumn{2}{|c|}{$0 / 10$} & \multicolumn{2}{|c|}{$0 / 9$} \\
\hline Lipid medication before study & \multicolumn{2}{|c|}{$5 / 5$} & \multicolumn{2}{|c|}{$4 / 5$} \\
\hline
\end{tabular}

CRP, C-reactive protein; eGFR, estimated glomerular filtration rate.

* Mean values were not significantly different between the two groups (resveratrol $v$. placebo) in the listed parameters. $\dagger$ eGFR was calculated by the Cockroft-Gault formula. 


\section{Results}

The study was completed with the full compliance of all patients enrolled. The patients who were randomly assigned to the resveratrol and placebo groups initially formed an apparently homogeneous population regarding all the assessed parameters (Table 1), even considering their previous lipid medication usage. Significant differences between the two groups in the assessed clinical and biochemical parameters surfaced at the end of the fourth week of the trial (week 4).

Fig. 1(a) shows a typical example of resveratrol-treated patients' CGM records after consuming the test meal at baseline and at week 4. Some noticeable differences between the two groups (i.e. placebo group $v$. resveratrol group) emerged at week 4 . While the records of placebo group at both baseline and week 4, and those of the resveratrol group at baseline were virtually indistinguishable (not shown), significant differences were found, when these records were statistically analysed for either the two groups (i.e. placebo group $v$. resveratrol group) at week 4 or the resveratrol group at baseline $v$. week 4 . For instance, the CGM records of the resveratrol group at week 4 displayed a distinct initial drop followed by a recovery phase (see Fig. 1(a)), which was reflected in two statistically analysable parameters. First, the time to the maximum glucose level significantly differed within the resveratrol group at baseline $v$. week 4 (49.50 (SD 13.83) v. 81.25 (SD 20.49) min, $P=0.006$ ), as well as between the two groups at week 4 (81.25 (SD 20.49) v. 58.1 (SD 18.42) $\mathrm{min}, P=0.03$ ). Meanwhile, the time to the maximum glucose appearance in the placebo group did not significantly differ at baseline $v$. week 4 (42.00 (SD 20.19) v. 56.43 (SD 18.42) $\mathrm{min}, P=\mathrm{NS}$; Fig. 1(b)). Second, as shown in Fig. 1(c), the extent of the initial drop (between 23 and $35 \mathrm{~min}$ after the test meal) was again significantly different between the two groups at week 4 (6.79) (SD 2.95) v. 8.64 (sD 4.42) $\mathrm{mmol} / \mathrm{l}, P=0.023)$.

No difference in serum insulin levels between the resveratrol and the placebo groups was observed at any time during the trial (i.e. at baseline $v$. week 2 or 4 ).

Because of the large individual variations in the measured $\mathrm{HOMA}_{\mathrm{IR}}$ values within the groups, a statistical comparison of the groups showed only a tendency of decrease upon resveratrol treatment at week 4 but with no statistical significance (i.e. $P=0 \cdot 112$; not shown). However, as shown in Fig. 2, if the changes in $\mathrm{HOMA}_{\mathrm{IR}}$ values at week $4 v$. baseline on the level of individuals were compared, then the differences were averaged within the respective group, a significant dissimilarity between the two groups emerged (resveratrol group: $-1.52(\mathrm{SD} 1.18) \quad v$. placebo group: 0.04 (SD 1.4), $P=0 \cdot 044)$. No differences were observed for $\mathrm{HOMA}_{\beta}$ values (data not shown).

The test values of the urinary $o$-Tyr:creatinine ratio, which were averaged within the respective group, showed a noticeable decrease for the resveratrol group at week 4 , which, however, was not significant probably due to the extremely large individual variations among the participants (not shown). On the other hand, a clearly significant difference between the two groups again surfaced (0.02 (SD 0.046) v. - 0.015 (SD $0.014) \mu \mathrm{mol} / \mathrm{mmol}, P=0.043$; Fig. 3), if the values of difference (i.e. the week 4 -baseline) on the individual level were considered.

Fig. 4 illustrates that a 4 -week-long treatment with resveratrol significantly increased the ratio of phosphorylated $v$. total Akt (pAkt:Akt) ratio in platelets (0.78 (SD 0.25) v. 1.41 (sD 0.36), $P=0.032$ ), while the pAkt:Akt ratio in the placebo group did not change during the trial (0.81 (SD 0.54) v. 0.72 (SD 0.37)).

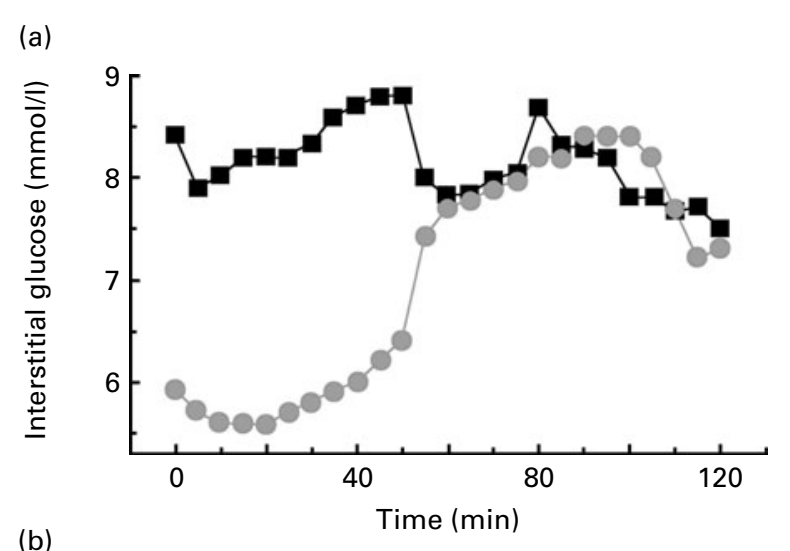

(b)

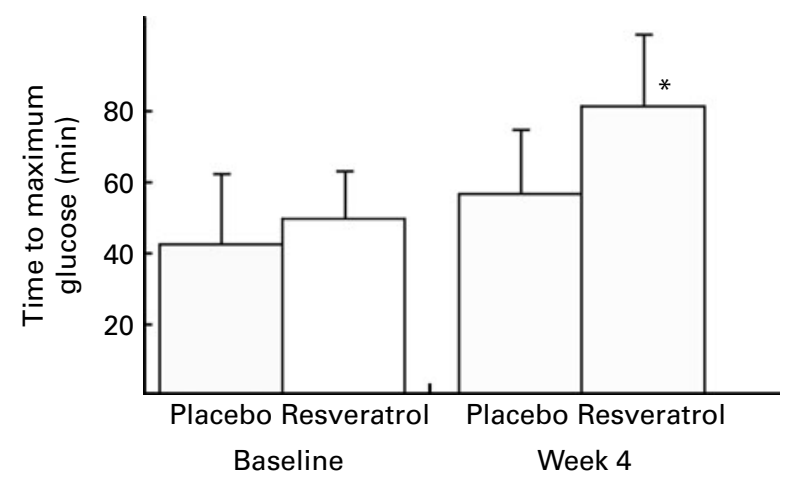

(c)

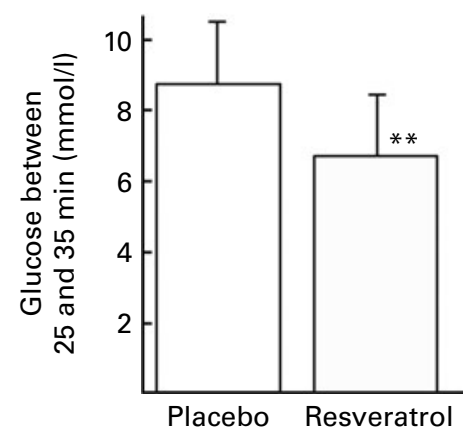

Fig. 1. Effect of resveratrol treatment on continuous glucose monitoring records. (a) An example before and after resveratrol treatment. ( $\mathbf{\square}$, Baseline; - week 4). (b) Statistical analysis of time until maximum interstitial glucose is reached after the test meal in the placebo and resveratrol groups. (c) Glucose levels at 25-35 min after the test meal (at week 4) in the placebo and resveratrol groups. Values are means, with standard deviations represented by vertical bars. * Mean values were significantly different for baseline $v$. week 4 within the resveratrol group $(P=0.006)$. ${ }^{\star *}$ Mean values were significantly different for the resveratrol group $v$. placebo group $(P=0.023)$. 
No significant changes were found for amylin, GIP and GLP-1 throughout the trial. At week 4, the amylin level was 2.98 (SD 0.69) pM in the resveratrol group and 3.11 (SD $2.93) \mathrm{pm}$ in the placebo group (NS); the GIP level was 38.22 (SD 9.44) $\mathrm{pg} / \mathrm{ml}$ in the resveratrol group and 36.23 (SD $8 \cdot 17) \mathrm{pg} / \mathrm{ml}$ in the placebo group (NS); and the GLP-1 level was 7.73 (SD 1.93) pm in the resveratrol group and 7.21 (SD $0.72)$ pm in the placebo group (NS).

However, we noticed that the initial negative correlation between systolic hyperbaric impact and ISI Stumvoll $_{\text {disappeared }}$ as a result of resveratrol treatment $\left(R_{\mathrm{visit} 1}-0.826, P=0.003 v\right.$. $R_{\text {visit } 3}-0.028, P=0.943$ for the resveratrol group $)$ and $\left(R_{\text {visit } 1}\right.$ $-0.757, P=0.029 v \cdot R_{\text {visit } 3}-0.716, P=0.046$ for the placebo group). Similar results were obtained, when the systolic hyperbaric impact was correlated with $\mathrm{MCR}_{\text {stumvoll: }}\left(R_{\text {visit } 1}\right.$ $-0.834, P=0.003 v \cdot R_{\text {visit } 3}-0.023, P=0.953$ for the resveratrol group) and $\left(R_{\mathrm{visit} 1}-0.757, P=0.029 v \cdot R_{\mathrm{visit} 3}-0.717, P=0.045\right.$ for the placebo group), respectively.

\section{Discussion}

In the present double-blind, placebo-controlled study, we tested the effects of trans-resveratrol on male patients with type 2 diabetes, and found that oral resveratrol in reasonably low dosages $(2 \times 5 \mathrm{mg}$ daily) improved insulin resistance and, probably as a consequence, decreased blood glucose levels and delayed the appearance of glucose peaks after a test meal. On the other hand, no resveratrol-induced changes were found in GLP-1, GIP and amylin levels. In addition, we also found that resveratrol seemed to decrease oxidative stress, as assessed by measuring urinary $o$-Tyr excretion, and increase Akt phosphorylation. At the same time, we found no evidence that resveratrol would affect $\beta$-cell function $\left(\mathrm{HOMA}_{\beta}\right)$.

The present findings appear to be in accordance with the results of previous studies, which have suggested an antioxidant role for resveratrol. For instance, resveratrol has previously been found to lessen oxidative stress in isolated K562 human leukaemia cells ${ }^{(25)}$ and in rats with streptozocin-induced diabetes ${ }^{(17,18)}$, which might relate to resveratrol's

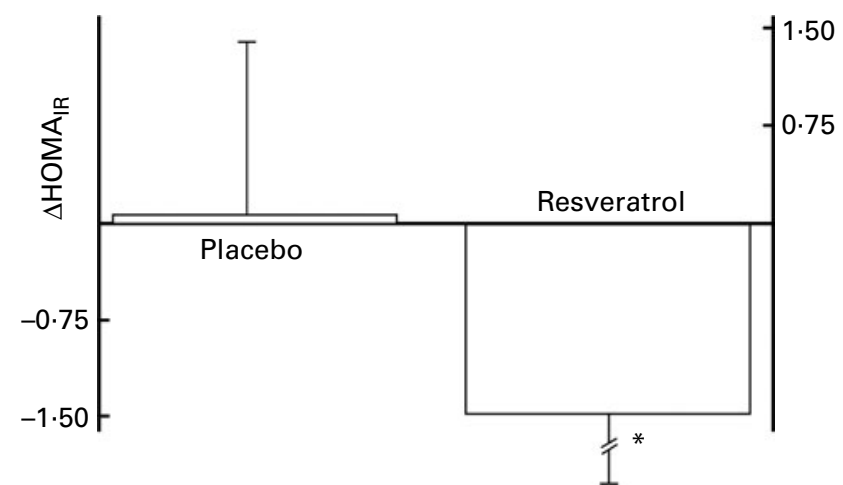

Fig. 2. Decrease in insulin resistance homeostasis model of assessment for insulin resistance $\left(\mathrm{HOMA}_{\mathrm{IR}}\right)$ upon resveratrol treatment. Values are means between baseline and week 4 for the placebo and resveratrol groups, with standard deviations represented by vertical bars. * Mean values were significantly different for the resveratrol group $v$. the placebo group $(P=0.044)$.

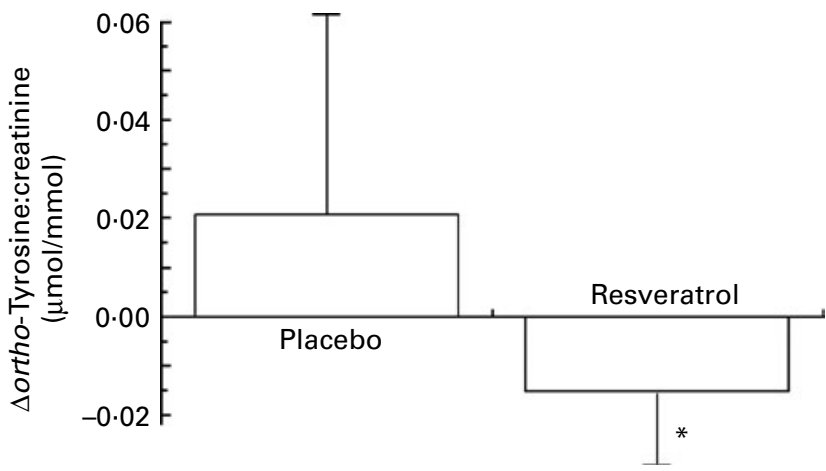

Fig. 3. Changes in urinary ortho-tyrosine:creatinine excretion after resveratrol treatment. The test values measured at week 4 were subtracted from those at baseline for each participant separately. Values are means, with standard deviations represented by vertical bars. ${ }^{*}$ Mean values were significantly different $(P=0.043)$.

potential to scavenge oxygen free radicals in vitro ${ }^{(15)}$. On the other hand, it also seems to be capable of both decreasing the production of oxygen free radicals and increasing certain antioxidant enzyme levels/activity ${ }^{(26)}$. In this regard, it is interesting to note that a simple calculation that considers the amounts of the excreted $o-\mathrm{Tyr}$ and the effective resveratrol dosage applied would suggest that the 'antioxidant' effect of resveratrol observed in the present study might not be due to a direct free radical-scavenging mechanism.

It has previously been shown that a negative correlation exists between hypertension and insulin sensitivity (i.e. ISI $_{\text {Stumvoll }}{ }^{(27)}$ ). Thus, it is worth noting that the negative correlation between systolic hyperbaric impact, ISI Stumvoll $_{\text {and }}$ $\mathrm{MCR}_{\text {Stumvoll }}$ seemed to disappear upon resveratrol treatment, suggesting that resveratrol might affect the nature of the association between insulin resistance and hypertension, which itself seems to warrant further studies on its vascular effects in relation to its influence on glucose regulation.

The blood glucose-lowering effect of resveratrol in diabetic rats has been documented and found to be associated with a resveratrol-induced activation of Akt and endothelial NO

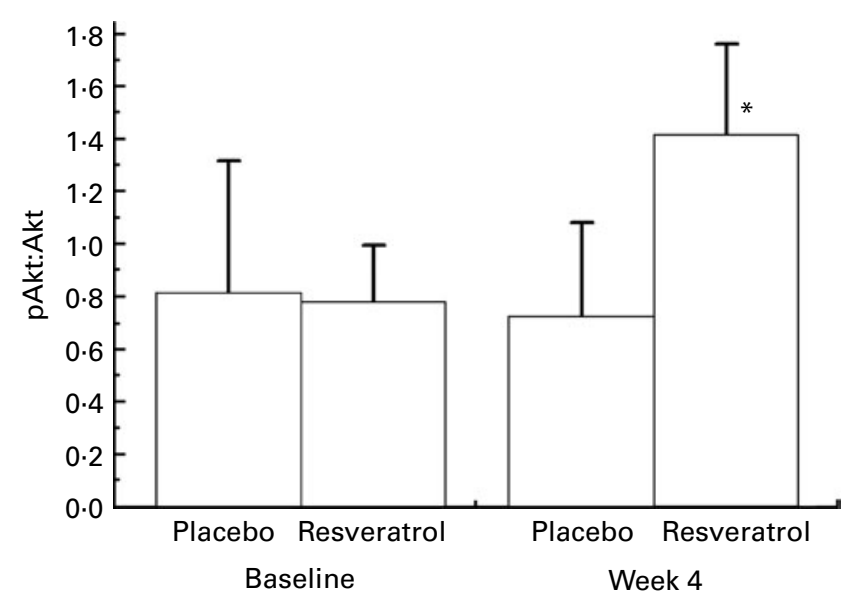

Fig. 4. Increase in protein kinase B phosphorylation (pAkt) in platelets upon resveratrol treatment. Values are means, with standard deviations represented by vertical bars. ${ }^{*}$ Mean values were significantly different for baseline $v$. week 4 within the resveratrol group $(P=0.032)$. 
synthase $^{(28,29)}$. The phosphorylation of Akt is known to be an essential step of insulin signalling ${ }^{(30)}$. The present findings with human subjects support the notion that the insulin resistance-lowering effect of resveratrol also occurs via the activation of the Akt signalling pathway. On the other hand, oxidative stress is now widely accepted to be a part of the aetiology of insulin resistance ${ }^{(13,14)}$. The present results seem to suggest that, in parallel with reducing insulin resistance, resveratrol might decrease oxidative stress. Although at this time it is not possible to pinpoint a clear cause-and-effect relationship between oxidative stress and some impairment in Akt-activating mechanisms, it is tempting to speculate that such causative coupling might exist.

Whether resveratrol (or some of its future derivatives) becomes a useful tool in combating type 2 diabetes is difficult to tell, although the fact that recent studies ${ }^{(31,32)}$ (including the present study) have demonstrated that the efficacy of resveratrol at low doses might increase the possibility for its medicinal application. On the other hand, the present study definitely suggests that resveratrol could become a useful tool in gaining a deeper understanding of the mechanisms underlying the development of insulin resistance and oxidative stress.

\section{Acknowledgements}

The present study received no specific grant from any funding agency in the public, commercial or not-for-profit sectors. P. B. organised the clinical visits, sample collection, statistical analysis and manuscript preparation. G. A. M. made the CGM system measurements, and helped in the statistical analysis and manuscript preparation. M. M. helped in the CGM system measurements and manuscript preparation. L. M. measured the oxidative stress markers (HPLC measurements) and helped in the manuscript preparation. B. L. performed the Western blotting experiments and helped in the manuscript preparation. J. C. helped in the Western blotting experiments and manuscript preparation. E. M. isolated platelets from blood samples for Western blotting and helped in the manuscript preparation. I. A. S. helped in the measurement of oxidative stress markers and manuscript preparation. Á. M. helped in the HPLC measurements and manuscript preparation. R. H. randomised the cases and controls and helped in the preparation of figures. L. G. M. helped in the study design, statistical analysis and participated in manuscript preparation. B. S. made the ELISA measurements, and was involved in the study design and application for funding. I. W. contributed to the idea generation and coordination of the study. There is no conflict of interest associated with the present study.

\section{References}

1. Renaud S \& de Lorgeril M (1992) Wine, alcohol, platelets, and the French paradox for coronary heart disease. Lancet 339, 1523-1526.

2. Goldfinger TM (2003) Beyond the French paradox: the impact of moderate beverage alcohol and wine consumption in the prevention of cardiovascular disease. Cardiol Clin 21, $449-457$.
3. Cucciolla V, Borriello A, Oliva A, et al. (2007) Resveratrol: from basic science to the clinic. Cell Cycle 6, 2495-2510.

4. Silan C (2008) The effects of chronic resveratrol treatment on vascular responsiveness of streptozotocin-induced diabetic rats. Biol Pharm Bull 31, 897-902.

5. Ray PS, Maulik G, Cordis GA, et al. (1999) The red wine antioxidant resveratrol protects isolated rat hearts from ischemia reperfusion injury. Free Radic Biol Med 27, 160-169.

6. Palfi A, Bartha E, Copf L, et al. (2009) Alcohol-free red wine inhibits isoproterenol-induced cardiac remodeling in rats by the regulation of Akt1 and protein kinase $\mathrm{C}$ alpha/beta II. J Nutr Biochem 20, 418-425.

7. Wallerath T, Deckert G, Ternes T, et al. (2002) Resveratrol, a polyphenolic phytoalexin present in red wine, enhances expression and activity of endothelial nitric oxide synthase. Circulation 106, 1652-1658.

8. Poussier B, Cordova AC, Becquemin JP, et al. (2005) Resveratrol inhibits vascular smooth muscle cell proliferation and induces apoptosis. J Vasc Surg 42, 1190-1197.

9. Radnai B, Tucsek Z, Bognar Z, et al. (2009) Ferulaldehyde, a water-soluble degradation product of polyphenols, inhibits the lipopolysaccharide-induced inflammatory response in mice. J Nutr 139, 291-297.

10. Delbosc S, Paizanis E, Magous R, et al. (2005) Involvement of oxidative stress and NADPH oxidase activation in the development of cardiovascular complications in a model of insulin resistance, the fructose-fed rat. Atherosclerosis 179, 43-49.

11. Houstis N, Rosen ED \& Lander ES (2006) Reactive oxygen species have a causal role in multiple forms of insulin resistance. Nature 440, 944-948.

12. Wei Y, Whaley-Connell AT, Chen K, et al. (2007) NADPH oxidase contributes to vascular inflammation, insulin resistance, and remodeling in the transgenic (mRen2) rat. Hypertension 50, 384-391.

13. Evans JL, Maddux BA \& Goldfine ID (2005) The molecular basis for oxidative stress-induced insulin resistance. Antioxid Redox Signal 7, 1040-1052.

14. Baynes JW (1991) Role of oxidative stress in development of complications in diabetes. Diabetes 40, 405-412.

15. Rüweler M, Gülden M, Maser E, et al. (2009) Cytotoxic, cytoprotective and antioxidant activities of resveratrol and analogues in C6 astroglioma cells in vitro. Chem Biol Interact 182, $128-135$.

16. Bhavnani BR, Cecutti A, Gerulath A, et al. (2001) Comparison of the antioxidant effects of equine estrogens, red wine components, vitamin $\mathrm{E}$ and probucol on low-density lipoprotein oxidation in postmenopausal women. Menopause 8, 408-419.

17. Sharma S, Anjaneyulu M, Kulkarni SK, et al. (2006) Resveratrol, a polyphenolic phytoalexin, attenuates diabetic nephropathy in rats. Pharmacology 76, 69-75.

18. Kumar A, Kaundal RK, Iyer S, et al. (2007) Effects of resveratrol on nerve functions, oxidative stress and DNA fragmentation in experimental diabetic neuropathy. Life Sci 80, 1236-1244.

19. Cockcroft DW \& Gault MH (1976) Prediction of creatinine clearance from serum creatinine. Nephron 16, 31-41.

20. Nagaretani H, Nakamura T, Funahashi T, et al. (2001) Visceral fat is a major contributor for multiple risk factor clustering in Japanese men with impaired glucose tolerance. Diabetes Care 24, 2127-2133.

21. Matthews DR, Hosker JP, Rudenski AS, et al. (1985) Homeostasis model assessment: insulin resistance and B-cell function from fasting plasma glucose and insulin concentrations in man. Diabetologia 28, 412-419. 
22. Stumvoll M, Mitrakou A, Pimenta W, et al. (2000) Use of the oral glucose tolerance test to assess insulin release and insulin sensitivity. Diabetes Care 23, 295-301.

23. Molnár GA, Wagner Z, Markó L, et al. (2005) Urinary orthotyrosine excretion in diabetes mellitus and renal failure: evidence for hydroxyl radical production. Kidney Int $\mathbf{6 8}$, 2281-2287.

24. Wagner L, Laczy B, Tamaskó M, et al. (2007) Cigarette smoke-induced alterations in endothelial nitric oxide synthase phosphorylation: role of protein kinase C. Endothelium 4, 245-255.

25. Chan WH (2005) Effect of resveratrol on high glucoseinduced stress in human leukemia K562 cells. J Cell Biochem 94, 1267-1279.

26. Yen GC, Duh PD \& Lin CW (2003) Effects of resveratrol and 4-hexylresorcinol on hydrogen peroxide-induced oxidative DNA damage in human lymphocytes. Free Radic Res 37, 509-514.

27. Kanauchi M, Kimura K, Akai Y, et al. (2004) Insulin resistance and pancreatic beta-cell function in patients with hypertensive kidney disease. Nephrol Dial Transplant 19, 2025-2029.

28. Thirunavukkarasu M, Penumathsa SV, Koneru S, et al. (2007) Resveratrol alleviates cardiac dysfunction in streptozotocininduced diabetes: role of nitric oxide, thioredoxin, and heme oxygenase. Free Radic Biol Med 43, 720-729.

29. Rivera L, Morón R, Zarzuelo A, et al. (2009) Long-term resveratrol administration reduces metabolic disturbances and lowers blood pressure in obese Zucker rats. Biochem Pharmacol 77, 1053-1063.

30. Avogaro A, de Kreutzenberg SV \& Fadini GP (2008) Oxidative stress and vascular disease in diabetes: is the dichotomization of insulin signaling still valid? Free Radic Biol Med 44, 1209-1215.

31. Baur JA \& Sinclair DA (2006) Therapeutic potential of resveratrol: the in vivo evidence. Nat Rev Drug Discov 5, 493-506.

32. Perdew GH, Hollingshead BD, Dinatale BC, et al. (2010) Estrogen receptor expression is required for low-dose resveratrol-mediated repression of aryl hydrocarbon receptor activity. J Pharmacol Exp Ther 335, 273-283. 